\title{
Práticas de gestão da informação e do conhecimento em pequenas e médias empresas organizadas em rede: um estudo multicasos na indústria brasileira
}

Ricardo Vinícius Dias Jordão

Professor do Mestrado em Administração da Fundação Pedro Leopoldo, Brasil.. Pesquisador do Center for Advanced Studies in Management and Economics (UE), Portugal

http://dx.doi.org/10.1590/1981-5344/1737

A pesquisa descrita neste artigo teve como objetivo analisar a influência da organização em rede sobre as práticas de gestão da informação e do conhecimento de pequenas e médias empresas (SMEs, do inglês small and medium-sized enterprises) industriais brasileiras. Através de um estudo comparativo de casos de abordagem qualitativa entre dois seguimentos industriais, agronegócios e indústrias em geral, concluiu-se que: (1) os processos de criação, aquisição, manutenção $e$ compartilhamento de informações e conhecimentos foram influenciados pela rede, bem como pela cultura organizacional, pela cooperação e colaboração, e pelas relações e interações existentes dentro e entre as empresas, (2) a troca de informações e experiências traz benefícios para as SMEs (3), a rede promoveu em parte a sistematização do conhecimento, mas facilitou o seu desenvolvimento, (4) o estabelecimento de um contexto favorável ('Ba') pode facilitar a criação e o desenvolvimento das habilidades desses indivíduos e empresas. Nesse sentido, observou-se que a cultura de cooperação pode ser considerada essencial no processo de aprendizagem individual e organizacional.

Palavras-chave: Gestão do Conhecimento; Gestão da Informação; Indústria Brasileira; Redes Interorganizacionais e de Conhecimento; Pequenas e Médias Empresas. 


\title{
Knowledge and information management practices in small and medium-sized enterprises organized in cooperative networks: a multi case comparative study in the Brazilian industry
}

\begin{abstract}
The research described in this article aims to analyse the influence of networking on information and knowledge management practices of small and medium enterprises (SMES) of the Brazilian industry. Through an eight comparative case study of qualitative approach, between two industrial segments, agribusinesses and industries in general, it was concluded that: (1) the processes of creation, acquisition, maintenance and sharing of information and knowledge were influenced by the network as well as by organizational culture, cooperation and collaboration, and the existing relations and interactions within and between enterprises, (2) the exchange of information and experience is beneficial for SMEs (3), the network promoted partly the systematization of knowledge, but facilitated its development, (4) the establishment of an enabling context ('Ba') can facilitate the creation and the development of skills of these individuals and companies. Accordingly, it was found that the culture of cooperation may be considered essential in the organizational and individual learning process.
\end{abstract}

Keywords: Knowledge Management; Information Management; Brazilian Industry; Inter organizational and Knowledge Networks, Small and Medium-sized Enterprises (SMES).

Recebido em 31.04.2014 Aceito em 20.09.2015

\section{Introdução}

A temática que envolve a gestão da informação e do conhecimento (GIC) tem ganhado espaço na literatura contemporânea (CHOO; ALVARENGA NETO, 2010; JORDÃO et al., 2013), sendo considerado um tema de grande interesse nas comunidades acadêmicas e no ambiente empresarial. Alguns autores (e.g. TOMAÉL, 2005; PELUFÊ, 2005; 
VALKOKARI; HELANDER, 2007; BEHR; NASCIMENTO, 2008; JARDON; MARTOS, 2012, 2014) têm ressaltado que o compartilhamento da informação consiste na questão essencial para melhorar a capacidade das organizações de gerar valor e de fomentar a aprendizagem organizacional. Choo e Alvarenga Neto (2010) ponderam que uma adequada GC colabora para que as empresas possam criar e manter vantagens competitivas sustentáveis nos setores em que operam, lembrando que a GC passa por questões como a criação, o uso e o compartilhamento de informações e conhecimentos, assim como pela criação do contexto capacitante 'Ba' que se refere às condições que devem ser propiciadas pelas empresas para se utilizarem melhor da informação e do melhor conhecimento disponíveis.

Segundo Tomaél (2005), a cooperação entre as empresas e pessoas é fundamental na construção do saber organizacional, facilitando que os ativos sejam utilizados em redes de conhecimento - ambientadas no seu contexto socioeconômico, cultural e nos processos de trabalho. As premissas dessa autora de (i) que o relacionamento visando à cooperação tornou-se o ponto central da nova forma organizacional e de (ii) que os processos de compartilhamento da informação e de construção do conhecimento nas redes possuem um papel central nos empreendimentos modernos são a base da pesquisa ora descrita.

Mesmo considerando a importância estratégica de micro, pequenas e médias empresas (SMEs, do inglês small and medium enterprises) para a economia (e.g. DURST; EDVARDSSON, 2012), as sociedades (e.g. PELUFÊ, 2005; KHALIQUE; ISA, 2014) e os governos (e.g. KHALIQUE; ISA, 2014), o desafio de compreender as peculiaridades da GC nas SMEs organizadas em redes é um fato que precisa ser mais bem investigado, pois, conforme Valkokari e Helander (2007), ainda se sabe pouco sobre o tema.

Reconhecendo e aproveitando essa lacuna de pesquisa, o objetivo da pesquisa ora descrita consistiu em analisar a influência da organização em rede sobre as práticas de gestão da informação e do conhecimento em SMEs industriais brasileiras.

A justificativa de tal pesquisa se dá na medida em que seus resultados contribuem para um segmento substancial da sociedade, como defendido por Jordão e Souza (2013) e Jordão et al. (2014). Pelufê (2005) lembra a crescente relevância que cerca o tema da GC no contexto das SMEs - que, paradoxalmente, têm sofrido tanto com o excesso de dados e com a falta de informações e conhecimentos úteis, quanto com a tradução desses fatores em decisões estratégicas. Além disso, 0 assunto pesquisado consta com as recomendações de estudiosos como Pelufê (2005), Valkokari e Helander (2007), Behr e Nascimento (2008), Durst e Edvardsson (2012), dentre outros. Assim, buscou-se trazer contribuições (i) teóricas (por aumentar o entendimento do efeito da organização em redes sobre as práticas de GC em SMEs - entendimento esse ainda incipiente na literatura, especialmente na realidade brasileira e no setor 
industrial) e (ii) empíricas (por melhorar o entendimento das práticas de compartilhamento de informações e conhecimentos em redes de SMEs).

Este artigo está dividido em mais sete seções para além desta introdução. Na seção 2 discute-se a gestão de informações e conhecimentos no contexto atual. Na seção 3 analisam-se as peculiaridades da GC em redes de SMEs. Na seção 4 descreve-se a metodologia da pesquisa. Na seção 5 apresenta-se uma contextualização do projeto e das empresas. Na seção 6 são apresentados e analisados os resultados do estudo. Por fim, na seção 7 tecem-se considerações finais à luz dos objetivos iniciais propostos, seguidas das referências bibliográficas utilizadas.

\section{Gestão da Informação e do Conhecimento}

Ao longo das últimas décadas, muitas partes da economia global têm se mudado para uma plataforma sustentada no conhecimento em que a criação de riqueza está associada ao desafio de desenvolvimento e gestão dos ativos intangíveis formadores do capital intelectual (CI) (KHALIQUE; ISA, 2014). Os intangíveis vêm crescendo em importância em função da potencialidade de geração de caixa, contribuição para o resultado econômico e representatividade de tais ativos para as empresas (JORDÃO, 2011; JORDÃO; COLAUTO, 2013). Adicionalmente, Ngah e Ibrahim (2009) elucidam que com a nova direção em que a economia está se movendo, informações valorosas estão fluindo de muitas fontes e canais, sem qualquer limitação.

Pelufê (2005) entende que, ao assumir o foco de grande parte das mudanças do século XX, a informação passou a ser objeto de estudo e alvo de distintas conceituações. Entendendo que a informação assume o papel de apoio à tomada de decisão e não se confunde necessariamente com 'dado' e 'conhecimento', ela é tida como recurso a ser utilizado pelos executivos a fim de ampará-los na redução das incertezas presentes no ambiente organizacional. Segundo Davemport e Prusak (1998) dados são definidos como um conjunto de fatos distintos e objetivos relativos a eventos que, quando trabalhados agregando-lhes valor, passam a constituir uma informação. No entanto, Pelufê (2005) e Ngah e Ibrahim (2009) ponderam que para esse processo de agregação de valor acontecer entre a informação e o conhecimento é necessário que haja tanto um trabalho de conexão e análise entre as informações, quanto um processo de socialização de umas com outras pessoas para a validação do conhecimento. Esses autores ressaltam que o conhecimento possui algumas características próprias, como 0 fato de ser difundível, substituível, transportável e compartilhável.

Segundo Hoss (2003), a informação torna-se inútil sem o conhecimento do ser humano para aplicá-la produtivamente, sendo a transferência desse conhecimento feita por duas maneiras: pela informação ou tradição. A informação é um tipo de comunicação utilizando a linguagem para articular alguns conceitos, na tentativa de transferi-los a 
outras pessoas - indicada para transmitir o conhecimento explícito. Já a transferência de conhecimento pela tradição se dá em conversas informais e de forma não estruturada - indicada para transmitir o conhecimento tácito.

Behr e Nascimento (2008) elucidam que a forma de gerenciar o conhecimento deveria ser percebida a partir do ambiente no qual este se desenvolve e a partir dos efeitos que ela exerce e sofre desse ambiente. Esses autores ressaltam que uma adequada GC tem de diferenciar o conhecimento tácito do explícito, sendo o primeiro não documentado e quase não passível de ensino e difícil de ser externalizado e o segundo, observável e documentável, esquemático, 'simples' e passível de ensino.

O processo de conversão de conhecimento é definido por Nonaka et al. (2000) como o processo pelo qual as organizações criam conhecimento e facilitam que o conhecimento tácito e explícito seja expandido qualitativa e quantitativamente. Existem quatro modos de conversão de conhecimento: (1) socialização (conversão de parte do conhecimento tácito de uma pessoa para outra), (2) externalização (explicitação de parte do conhecimento tácito do indivíduo), (3) combinação (conversão de algum tipo de conhecimento explícito individual em explícito organizacional) e (4) internalização (internalização de partes do conhecimento explícito da organização em conhecimento tácito individual). Teoricamente, os chamados ciclos de conversão do conhecimento, passando várias vezes por esses quatro modos, formariam uma espiral que serve para analisar e entender os mais diversos casos de criação e disseminação do conhecimento.

\section{Gestão do Conhecimento em Redes de SMEs}

Nesse novo contexto concorrencial em que atuam as SMEs (caracterizado por uma maior interligação com o ambiente), as redes despontam como alternativa para que essas empresas tenham maior competitividade e mais acesso a produtos, serviços, tecnologias e mercados, como explica Castells (1999). A literatura internacional (e.g. ROMITI; SARTI, 2011; JARDON; MARTOS, 2014) reconhece que é a partir da atuação conjunta e da cooperação entre SMEs, com o objetivo de se tornarem mais eficientes e competitivas, que surge a lógica das mesmas atuarem em rede - compartilhando informações e conhecimentos.

A realização de ações conjuntas entre as empresas parceiras favorece a criação e conversão de conhecimentos, gerando CI e inovação (CALDAS; CÂNDIDO, 2013), além de proporcionar melhorias no desempenho das mesmas (KHALIQUE et al., 2015). Autores como Nonaka e Nishiguchi (2001) e Spencer (2003) também têm sinalizado a importância das relações interorganizacionais para o processo de emergência do conhecimento, argumentando que as redes podem ser mais efetivas que as formas de integração organizacional na criação, na transferência e na recombinação de conhecimento. Segundo Jordão et al. (2013) e Jordão et al. (2015), é nesse contexto que a GC se transforma 
em um valioso recurso estratégico para a vida das pessoas e das empresas na busca pela excelência humana, operacional e gerencial, facilitando o alcance dos objetivos estratégicos estabelecidos nos projetos empresariais e minimizando os riscos inerentes a eles.

Segundo Granoveter (1985), a presença no mesmo ambiente socioeconômico, a partilha de valores, costumes, rotinas, e a existência de uma vivência social estabelece uma gama de conhecimentos transmitidos não por códigos convencionais, mas, sim, pelo grau de imersão das empresas na rede. Sabel (1991) salienta que a confiança é central para o desenvolvimento das redes de SMEs - que tende a surgir a partir de uma estrutura ou de um contexto adequados. Para Suchman (1987), é preciso um contexto específico em termos de tempo, espaço e relacionamento entre indivíduos, a fim de que o processo de criação de conhecimento ocorra efetivamente ou socialização do mesmo. Já Caldas e Cândido (2013) recordam que o 'Ba' potencializa a geração e expansão qualitativa e quantitativa dos saberes tácito e explícito, assim como a unificação dos espaços físicos, virtuais e mentais, estimulando à socialização, externalização, combinação e internalização do conhecimento.

Barros et al. (2004) evidenciam que é necessário, para transformar o know-how individual em propriedade de um grupo, que os ativos intelectuais sejam estruturados como sistemas de informação, inteligência competitiva e de mercado, sistemas de conhecimentos dos canais de mercado e foco gerencial. Segundo Tomaél (2005), o conhecimento tácito é construído pelo compartilhamento de experiências e, por isso, é mais profícuo pela interação - que permite sua expansão, complemento e até aprimoramento - argumentando que as redes são fundamentais nesse processo. Essa autora analisou o compartilhamento de informações e conhecimentos em redes sociais de um consórcio de exportação de móveis, no norte do Estado do Paraná, concluindo que: (a) a informação é um importante elemento que incita à construção do conhecimento e inovação; (b) o processo de construção do conhecimento depende da interação espontânea dos atores; (c) as redes são consequência dessas interações - que se iniciam pelo compartilhamento de informação; (d) os atores que recebem mais informação, na rede, foram os mais influentes e os mais comprometidos; e (e) a rede despontou como um lugar propício ao compartilhamento de informações e conhecimentos, fortalecendo os projetos e processos empresariais.

Freeman et al. (2006) pesquisaram SMEs da Austrália e perceberam que elas se internacionalizam rapidamente quando utilizaram as redes e estabeleceram alianças estratégicas. Os resultados indicaram que essas empresas superam as limitações relacionadas ao seu porte por meio do uso de tecnologias e das redes para formar alianças e parcerias colaborativas. No caso brasileiro, Amal et al. (2008) pesquisaram o processo de internacionalização de SMEs, mostrando que as redes de relacionamentos e a existência de um perfil empreendedor influenciam na internacionalização dessas empresas. Já Silveira e Alperstedt (2007) estudaram a internacionalização de uma pequena organização 
empresarial. Os resultados indicaram que a internacionalização se deu com a ajuda da rede de relacionamentos do empreendedor e que a empresa se expandia à medida que ia obtendo novos conhecimentos.

Em suma, os resultados dessas pesquisas por vezes mostraram: (i) a relevância do compartilhamento de informações e conhecimentos no contexto das redes de SMEs; (ii) a necessidade da criação de um 'Ba' para que isso ocorra de maneira efetiva; (iii) que os relacionamentos entre os atores (pessoas e organizações) são essenciais nesse processo; e (iv) que as redes facilitam e estimulam tanto criação, sistematização e compartilhamento de informações e conhecimentos, quanto a aprendizagem pessoal e organizacional.

\section{Metodologia da pesquisa}

A pesquisa descrita neste trabalho, quanto à metodologia, pode ser classificada como um estudo comparativo de casos de natureza qualitativa e de abordagem explanatória (COOPER; SCHINDLER, 2003; GEORGE; BENNETT, 2005). O método de casos, de acordo com Yin (1984), consiste em uma investigação empírica de um fenômeno contemporâneo dentro de seu contexto da vida real, quando os limites entre fenômeno e contexto não estão claramente evidentes e várias fontes de evidências são utilizadas. Segundo Tomaél (2005), esse método é suficientemente sensível para analisar a complexidade inerente ao processo de compartilhamento de informações e conhecimentos no contexto das redes de empresas.

Dentre as várias fontes de evidências existentes em um estudo de caso de natureza qualitativa, priorizou-se, como fonte primária de evidências, seguir as recomendações de Eisenhardt (1989), realizando-se entrevistas pessoais, em profundidade, apoiadas em um roteiro de entrevista estruturado (com variáveis extraídas da literatura) contendo cinco questões abertas e quinze fechadas - que foi aplicado ao principal executivo de cada empresa (CEO, do inglês chief executive officer). As variáveis analisadas relacionam-se: (1) às habilidades e competências necessárias para o processo de exportação, a absorção, documentação, transmissão, retenção, criação e compartilhamento das informações, conhecimentos, experiências e competências desenvolvidas nas empresas e na rede; (2) às formas de relacionamento entre os atores; (3) a honestidade em compartilhar saberes e experiências (4) a forma de fomentar e reter conhecimento necessário ao processo de internacionalização; (5) ao ambiente físico e social para criação do conhecimento; (6) às ferramentas e ao processo de criação de métodos e partilha de linguagem; (7) aos incentivos para que o conhecimento individual e empresarial seja compartilhamento; (8) aos ganhos proporcionados pela rede aos participantes; (9) à forma de transmissão e partilha de conhecimentos sobre o processo de internacionalização; (10) ao intercâmbio de informações e interações formais e informais na rede; e 
(11) ao efeito de culturas similares, linguagem e experiência sobre a GC no interior da rede.

As questões fechadas visaram maior comparabilidade e as abertas buscaram proporcionar liberdade para explicações adicionais. As informações coletadas foram transcritas e depois tabuladas antes de serem analisadas. A maneira utilizada para investigar o significado simbólico e o conteúdo da comunicação linguística foi técnica de análise de conteúdo que, segundo Bardin (2004), auxilia a realização de ligações entre a situação a ser analisada e as manifestações da superfície discursiva. Conforme Jordão (2013) e Jordão e Novas (2013), isso se dá por meio de operações de desmembramento e classificação semânticas, sintáticas e lógicas.

O critério utilizado para se selecionar os casos estudados (e por consequência a rede e o Estado) foi o acesso às informações - seleção por tipicidade, segundo Cooper e Schindler (2003). A análise recaiu sobre uma rede de SMEs que receberam suporte técnico e de formação gerencial do Serviço Brasileiro de Apoio às Micro e Pequenas Empresas (SEBRAE) do Estado do Espírito Santo (ES). Dentre as 48 empresas pertencentes à rede, o levantamento inicial indicou que havia apenas seis empresas do setor agronegócios e sete do setor de indústrias em geral - das quais quatro de cada setor se dispuseram a participar da pesquisa (que serão tratadas por nomes fictícios em função de acordo de confidencialidade).

Visando aumentar a validade interna do estudo, procurou-se agregar informações coletadas em outras fontes de evidência (processo de triangulação) (JICK, 1979). Assim, sempre que possível, as informações advindas de uma fonte foram confrontadas com as de outras para confirmá-las e validá-las (JORDÃO, 2013). Esse processo iniciou-se com as próprias entrevistas, pois foram ouvidas pessoas diferentes sobre um mesmo tópico. Paralelamente, a triangulação envolveu a análise de folhetos informativos, de informações constantes em web pages, em manuais de procedimentos e em notícias publicadas em jornais e revistas sobre as empresas, o projeto ou os setores em que elas atuam (análise documental). Nesse sentido, optou-se por iniciar a análise a partir de uma apresentação contextualizada dos setores e da rede situando o projeto e as inter-relações de tais fatores com as empresas objetos do estudo. Por motivo de parcimônia, os resultados da triangulação foram apresentados ao longo do texto com as novas informações apresentadas, corroborando ou refutando as anteriores. Finalmente, analisaram-se também as informações obtidas em conversas informais (extra-entrevistas), além de contatos posteriores às entrevistas, com o propósito de complementar dados e de esclarecer as dúvidas existentes. Assim, seguindo os passos propostos por Eisenhardt (1989), buscou-se dar robustividade ao estudo e formar uma cadeia nomológica de evidências, mesclando informações internas e externas, além de confrontar os resultados entre si e confrontar estes com a literatura - o que se espera poder ajudar a compreender melhor o problema investigado. 


\section{Descrição contextualizada da Rede e das SMEs}

Com base em informações setoriais e em linha com as diretrizes do Ministério do Desenvolvimento, Indústria e Comércio Exterior, o SEBRAEES buscou fomentar as exportações das SMEs do ES - elaborando um projeto cujo público alvo consistia em SMEs por ele atendidas e que pretendiam exportar produtos e/ou serviços. Esse projeto foi concebido e executado com ajuda dos principais representantes do comércio exterior, identificando o potencial de cada setor e definindo os mecanismos de atuação que ajudassem a concretizar tal potencial. Dentre os setores contemplados estão os de agronegócios e de indústrias em geral, ora objetos de estudo. A análise documental indicou que esse projeto foi concebido com os objetivos de: (1) aumentar o número de SMEs exportadoras; (2) incrementar o volume e a qualidade dos negócios nessas empresas; (3) fomentar e consolidar a articulação e integração das SMEs do ES no mercado mundial; e (4) torná-las mais competitivas. Além disso, buscou-se (5) preparar as SMEs para serem mais competitivas no mercado doméstico (SEBRAE-ES, 2011). Inicialmente o projeto buscou transformar essas SMEs no aspecto da cultura organizacional (voltada para a internacionalização, a sustentabilidade e a atuação em rede), compartilhando informações e conhecimentos pelo e com o SEBRAE-ES e com as demais empresas da rede. Esse projeto tem contemplado (além dos cursos e treinamentos convencionais) capacitações gerenciais por meio de consultorias acopladas (com visitas in loco) nas SMEs selecionadas para aumentar a absorção dos conhecimentos tácitos e explícitos pelos membros dessas empresas, de maneira similar ao apontado por Suchman (1987).

O projeto buscou selecionar cem SMEs para atuarem no comércio internacional com sustentabilidade, mas apenas sessenta preencheram os requisitos (e destas 48 se mantiveram ativas). $\mathrm{Na}$ pesquisa, foram analisadas as seguintes empresas: ALFA, BETA, CAPA e DELTA, do setor de indústrias em geral; e GAMA, ZETA, SIGMA e ÔMEGA, do agronegócio. A classificação do porte das empresas veio do SEBRAE Nacional, tendo como base o número de empregados das mesmas, segundo o qual, todas as SMEs investigadas são indústrias de pequeno porte, salvo a empresa BETA - que é hoje uma empresa de médio porte.

No que concerne às indústrias em geral, a ALFA foi fundada a pouco mais de 20 anos e está localizada no município de Cachoeiro de Itapemirim ES, fabricando produtos e componentes para elevação e movimentação de materiais, beneficiamento de rochas e tratamentos de resíduos. A empresa se define como fabricante de equipamentos modernos e de alta durabilidade que procura acompanhar o contínuo desenvolvimento e surgimento de novas tecnologias, buscando trabalhar em uma filosofia de melhoria continua e inovar nos métodos de trabalho, produtos e processos para atender melhor as necessidades dos clientes. A análise documental revelou que, depois de sua integração ao projeto, essa empresa implantou um programa de qualidade total para melhor atender 
e satisfazer os clientes, visando o crescimento e o desenvolvimento da empresa, procurando a melhoria contínua, confirmando e ampliando os achados da literatura internacional de Romiti e Sarti (2011) e Jardon e Martos (2014), pois a atuação conjunta e a cooperação entre ela e as demais SMEs ajudaram-nas a se tornarem mais eficientes e competitivas, compartilhando informações e conhecimentos, desenvolvendo inovações e gerando valor aos stakeholders da empresa. A empresa BETA foi fundada há mais de 25 anos e, atualmente, consiste em um grupo empresarial formado por três diferentes empresas que se fundiram em 2010. Ela também está localizada na cidade de Cachoeiro de Itapemirim e fabrica equipamentos para diversos setores. De forma similar à ALFA essa empresa entende que o seu maior diferencial é o desenvolvimento de soluções customizadas, posicionando a empresa no segmento da inovação tecnológica fornecendo produtos de qualidade diferenciada e de fácil acesso para manutenção. A análise documental revelou que, depois do projeto, a BETA busca trabalhar agregando conhecimentos em seus produtos, alinhando sua estratégia mercadológica a uma sistemática de produção que controla desde as matérias primas até o pós-venda, confirmando, parcialmente, as premissas de Jordão et al. (2013) e Jordão et al. (2015). A empresa CAPA começou como uma empresa familiar em 1987, com sua sede industrial em Serra ES, onde produz biscoitos dietéticos e de polvilho para consumidores com alta exigência de qualidade. A empresa informa que, desde o início, a qualidade dos produtos e a preocupação com a saúde alimentar dos consumidores foram questões tidas como primordiais. A análise documental revelou que depois de sua integração à rede, essa empresa, além de manter e ampliar as linhas tradicionais, vem desenvolvendo novos produtos segundo as demandas do mercado, que "cada vez mais tem exigido produtos mais leves e saudáveis". Essa empresa, em linha com Pelufê (2005), vem buscando transformar informações e conhecimentos em decisões estratégicas, sendo que o projeto foi considerado importante nesse aspecto. A empresa DELTA foi fundada em 2001, estando localizada em Vila Velha ES. Ela atuava inicialmente na área de refugos e sucatas, depois no comércio de plásticos e finalmente na industrialização do mesmo. O CEO dessa empresa revela que sua integração à rede proporcionou alguns benefícios significativos, pois depois de participar de duas feiras na China e uma na África, a convite do SEBRAE, o empresário decidiu abrir em 2009/2010 uma empresa de importação, exportação e de acompanhamento de carga em Hong Kong e em 2011 abrir (com outros empreendedores) um centro de reciclagem e de fabricação de plástico em Moçambique. Ele explica que o negócio vem ganhando outros contornos ao revelar que "qualquer equipamento chinês que for comprado por um brasileiro, nós vamos testar e acompanhar desde a compra até a entrega do produto", corroborando e ampliando as observações de Andersen e Buvik (2002) e Silveira e Alperstedt (2007), pois tal empresa não somente usou a rede para se internacionalizar, mas também desenvolveu uma 
plataforma de conhecimentos que foram incorporados (internalizados e sistematizados) depois de sua integração à rede.

Relativamente às empresas do agronegócio, a GAMA foi fundada em 1989 para facilitar a organização dos produtores, a comercialização dos produtos e o estabelecimento de parcerias com institutos de pesquisa, além da identificação das características da região serrana do ES - onde a mesma se localiza. Foi observado pela análise documental que havia baixo valor agregado ao produto decorrente de baixa qualidade do mesmo e dos processos de fabricação. Observou-se que, com o passar do tempo, a GAMA veio buscando adequar a produção aos padrões internacionais de qualidade por meio de envolvimento dos produtores, orientações técnicas e novas tecnologias, readequação de produtos e processos, além de buscar (1) novas alternativas mercadológicas, (2) introdução de um café especial para venda no exterior, (3) estreitar suas relações com parceiros estratégicos, assim como (4) promover melhorias na gestão e organização dos associados. Depois do projeto a GAMA veio se conscientizando que a competição se dá em escala global e de que cresce, a cada dia, a exigência pela rastreabilidade e qualidade dos produtos, como proposto por Choo e Alvarenga Neto (2010), Pelufê (2005) e Behr e Nascimento (2008), relacionando melhorias na gestão com a competitividade e os processos de GC. A ÔMEGA está localizada a cerca de $100 \mathrm{~km}$ da capital, tentando produzir café segundo as práticas internacionais para aumentar a divulgação e aceitação de seu produto principal, comercializando-o juntamente com queijos, iogurtes, fubás de milho, linguiças e ricotas, além de explorar o agro turismo. A visão de negócio da empresa é primar pela qualidade dos produtos e pela satisfação dos clientes, inovando em produtos, serviços e processos - especialmente em cafés especiais. A análise documental indica que a família proprietária da empresa já vem aperfeiçoando a qualidade de seu café (da seleção das sementes até a degustação do café) desde a década de 1880 , quando chegou da Itália, resultando em premiação em concursos - o que deu uma maior visibilidade ao produto e abriu o caminho para exportação para Europa e Ásia, complementando as observações de Andersen e Buvik (2002) e Silveira e Alperstedt (2007). Já a SIGMA está localizada ao sul do ES em uma pequena cidade cuja economia é predominantemente rural. Nas décadas de 1920 e 1930, o café trouxe muita prosperidade para a região e ainda hoje é a principal atividade econômica do município. Além da comercialização de cafés de vários tipos, a SIGMA ainda tem como atividade econômica a armazenagem, o rebeneficiamento de café e a assistência técnica. Essa empresa tem uma estrutura de produção e negócios bastante similar a GAMA e, como aquela, esta vem investindo na modernização de suas máquinas e equipamentos e buscado financiamentos, parcerias, capacitação e certificações internacionais, dentre outras coisas. Diferentemente daquela, porém, a SIGMA estimula que haja mais espaços favoráveis à criação do conhecimento na sua estrutura organizacional ou nas inter-relações entre pessoas e/ou empresas, conforme defendido por Lei et al. (1996) e Nonaka e Nishiguchi 
(2001). Finalmente, a ZETA está posicionada (estrategicamente) às margens da BR 262 perto da divisa com Minas Gerais. A visão da empresa é explorar a posição estratégica do ES como segundo maior produtor de cafés do Brasil e como destaque internacional na produção de cafés especiais - que são muito valorizados no mundo. A análise documental indicou que a estratégia de marketing dessa empresa visa estimular os visitantes a experimentar uma culinária típica 'da roça', vendendo produtos alimentícios feitos artesanalmente e os cafés especiais. Em linha com o pensamento de Prahalad e Hamel (1990), a ZETA tem consciência de suas competências essenciais e vem desenvolvendo um programa de qualificação de sua produção, conseguindo, ao longo dos últimos anos, colher grãos de alta qualidade para a torrefação - o que permitiu a obtenção de um café considerado nas palavras dos proprietários "de um sabor incomparável, forte e encorpado". Cabe salientar que, nas propriedades da família, observou-se que a política e estratégia empresarial prezam por uma produção econômica, social e ambientalmente responsável e sustentável

\section{Apresentação e análise dos resultados}

Ao analisarmos comparativamente as empresas, observou-se que todas elas acreditam que possuem um bom nível de consciência sobre quais são os pontos fortes e fracos da empresa em termos de habilidades e competências necessárias para o processo de exportação, ampliando as observações de Choo e Alvarenga Neto (2010) enquanto necessidade da GC como ferramenta gerencial. Os entrevistados avaliaram que as empresas absorveram uma parte significativa do conhecimento construído na rede durante o projeto e, a exceção da CAPA, que o relacionamento existente entre as diferentes empresas da rede facilitou no processo de criação do saber, corroborando e ampliando o proposto por Tomaél (2005), Grant (1995), Mouritsen et al. (2002), Spencer (2003), Behr e Nascimento (2008) e Lei et al. (1996). Em especial, esses resultados mostram os efeitos da rede e dos relacionamentos dela decorrentes sobre o conhecimento formado dentro das empresas. Além disso, todos os empresários acreditam que as empresas da rede até conhecem alguns processos de negócios das outras empresas, aproveitando do intercâmbio de informações sobre as especificidades dos processos industriais e comerciais umas das outras, mas, metade deles, os executivos das empresas BETA, DELTA CAPA e GAMA, entendem que isso não se deu em quantidade e de maneira suficiente. Os executivos também, de maneira quase unânime, salvo CAPA e DELTA, percebem que os membros da rede têm interações que acontecem fora dos momentos formais estipulados pelo SEBRAE-ES para discussão das ideias inerentes ao projeto, mas vários deles pensam que tais momentos ainda são insuficientes para estreitar as relações das empresas pertencentes à rede, corroborando parcialmente as premissas de Tomaél (2005). Sobre essa questão, especificamente, eles acreditam que isso deveria ocorrer com maior 
intensidade e frequência, ressaltando uniformemente o que essa autora já havia afirmado: que o relacionamento existente entre as diferentes empresas da rede facilita no processo de criação do conhecimento.

Outras questões analisadas tiveram uma percepção menos uniforme por parte dos executivos. Na opinião do CEO da GAMA e da SIGMA não há investimentos e incentivos na formação profissional e pessoal dos membros das empresas envolvidas no projeto preparando-os para a exportação. Contrastando essa opinião, os executivos das demais empresas acreditam que eles existem. Todavia o CEO da ZETA pensa que o montante de investimentos aportado no projeto poderia ter sido superior ao que foi alocado e que o processo de formação não ocorreu exatamente da forma como isto deveria ser. Já o CEO da ÔMEGA mostrou-se satisfeito em relação a tal quesito. Por outro lado, contrariando os achados de Pelufê (2005), observou-se que quase todos (salvo os das empresas DELTA e ÔMEGA) parecem concordar que não há disciplina, eficiência e incentivo para a documentação do conhecimento e know-how desenvolvido na rede - sendo que apenas uma parte destes é absorvida no contexto das discussões e reuniões realizadas. Portanto, carecendo de maior sistematização e formalização no contexto das SMEs analisadas.

Uma das questões que os CEOs da CAPA, DELTA e GAMA ressaltam é o fato de que as empresas da rede ainda não desenvolveram um ' $B a^{\prime}$ para criação do conhecimento depois de se integrarem a rede. Os demais executivos concordam, em diferentes graus, que isso foi criado, em parte, mas que há bastante espaço para melhoria neste quesito, corroborando parcialmente e ampliando Suchman (1987) e Lei et al. (1996), pois eles constataram que a própria organização em rede consiste em um espaço para criação e compartilhamento de informações e saberes.

Há outras questões sobre as quais aqueles três CEOs divergem dos colegas das outras cinco. Uma delas é o fato de que os mesmos não perceberam a existência de ferramentas formais que ajudem a divulgar as práticas bem sucedidas de exportação dentro da rede, mas os demais perceberam sim, citando, como exemplos, as reuniões e os grupos de trabalho estabelecidos. Além disso, de um modo geral, os executivos da ZETA, ÔMEGA e SIGMA sentem que o projeto propiciou a criação de uma linguagem compartilhada, com métodos entendidos e utilizados pelos participantes da rede. Fato reconhecido, em parte, pelos CEOs da ALFA e BETA e pouco percebido pelos demais. Todos os executivos, contudo, verificaram o fato de que a rede possibilitou diferenciais competitivos para as empresas participantes, gerando, desse modo, os conhecimentos necessários para o processo de exportação, exceto o da GAMA que criticou o projeto dizendo que as "decisões tomadas nos dias dos workshops não foram cumpridas e não houve justificativas para o seu não cumprimento".

Ao contrário dos demais, os CEOs da BETA, CAPA, GAMA e ZETA parecem concordar que não há incentivos na rede para que 0 conhecimento individual seja compartilhamento com os outros membros da mesma e os três últimos entendem que o conhecimento prático não é 
passado para a rede em forma de conhecimento teórico e é novamente transformado em saber prático no momento de sua utilização - sendo que - CEO da GAMA criticou abertamente esta ideia. Ressalta-se, que as visões desses executivos eram diferentes. Enquanto o CEO da GAMA tinha uma visão negativa sobre o projeto e a organização em redes, os da CAPA, DELTA e ZETA tinham uma visão positiva, além de vislumbrarem um horizonte favorável. Esses três tinham consciência de que algumas das referidas questões poderiam até se manifestar para outras empresas de forma diferente, mas deixando claro que ainda careciam de grande aperfeiçoamento. Por outro lado, os CEOs da ALFA, BETA, SIGMA e ÔMEGA mostraram-se bastante satisfeitos com os resultados e com a estrutura do projeto, ponderando que ele tem proporcionado uma oportunidade de aprendizado e crescimento pessoal e profissional com significativo intercâmbio de informações e conhecimentos. Esses executivos notaram sim tais incentivos, destacando que a própria estrutura do projeto permitia tanto a absorção dos conhecimentos teóricos trazidos pelo SEBRAE-ES e seus associados, quanto o compartilhamento das experiências práticas das empresas, confirmando os preceitos técnicos e gerenciais observados no site e nos documentos institucionais do SEBRAE-ES. Esses resultados permitiram conclusões parciais sobre o ponto, com base em Pelufê (2005), mas ajudam a perceber que a absorção de conhecimentos e a partilha de experiências ocorrem de forma mais vinculada à empresa do que a rede e que o envolvimento das pessoas (a começar pelo CEO) é fundamental para o sucesso da GIC no âmbito das redes.

Segundo o CEO da GAMA, essas diferenças seriam porque há informações e conhecimentos não compartilhados por todos os membros e para e para todos eles, sugerindo que há empresas que conseguem maiores acessos às informações do que outras devido a seus relacionamentos, para além da rede, e que a rede não foi fundamental no processo de desenvolvimento das competências necessárias para a exportação na empresa dele. Contrastando essa visão, porém, os executivos das demais sete empresas analisadas perceberam, em diferentes graus, que a rede foi fundamental em ambos os processos e para a criação de uma cultura própria no interior do grupo (da rede) com algumas similaridades de identidade, linguagem e experiência compartilhadas - que eles entendem ajudar a criar e difundir 0 conhecimento no interior da rede.

Os CEOs da ALFA e ZETA ponderam, todavia, que há espaço para melhoria em todos estes quesitos e que ainda há muito a se fazer para que as empresas possam, de fato, criar, armazenar e compartilhar o conhecimento produzido em suas empresas e, que há ainda mais, para que isto se dê de forma efetiva no interior da rede. Refutando explicitamente, as ideias do CEO da GAMA, os colegas da BETA, DELTA, CAPA, SIGMA, ZETA e ÔMEGA indicam que, há, sim, honestidade intelectual das empresas que compõe a rede e que as pessoas têm certa autenticidade - deixando claro aquilo que conhecem e também o que não 
conhecem sobre as experiências adquiridas. Eles ponderam isto por perceber que (i) as informações são compartilhadas, mesmo que nem todas as empresas estejam no mesmo patamar das demais (carecendo de mais tempo para poderem atuar plenamente no mercado internacional); e que (ii) há acesso por parte dos membros à base de dados e conhecimento existentes nas outras empresas (mesmo que nem todos os membros à base de todas as empresas). Esses resultados ampliam o que se sabe sobre o processo de compartilhamento de informações e conhecimentos (iii) por mostrar que as redes são um espaço de aprendizagem organizacional, confirmado a premissa teórica de Lei et al. (1996), e (iv) por mostrar que o processo de partilha de informações e de fomento à aprendizagem organizacional pode ser percebido, realizado e trabalhado pelas empresas pertencente à rede de maneiras diferentes, indo além do que foi observado por Behr e Nascimento (2008) e Pelufê (2005). Extrapolando as conclusões de Granoveter (1985), esses resultados mostram também que os relacionamentos inter e intrarredes, bem como a similaridade cultural, foram entendidos por todos entrevistados, menos das empresas CAPA e DELTA, como promotores do compartilhamento de informações e conhecimentos no âmbito das redes que se formam não só no interior, como também no exterior das empresas e de suas conexões.

Buscando aumentar a validade interna do estudo, a triangulação com documentos revelou que todas as SMEs foram incentivadas pelo SEBRAE-ES a trabalhar de forma continuada para não haver interrupção do fluxo comercial e informacional proposto para o grupo, mostrando a necessidade de atuação em redes e orientando que os empresários absorvessem os termos técnicos e as ações executadas nas operações internacionais - visando capacitá-los a entender os mecanismos e processos a serem executados, bem como estimulá-los a usar e compartilhar informações e conhecimentos de forma a maximizar seu uso, gerando valor. Assim, as SMEs formaram uma rede de cooperação de conhecimentos, como observado por Tomaél (2005).

Ao realizar uma análise comparativa entre o setor de indústrias em geral e agronegócios, observou-se que não foram constatadas diferenças significativas entre os mesmos em relação às práticas de GIC, mas, sim, pelo grau de imersão das SMEs à rede, como preconizado por Granoveter (1985), pela cultura organizacional (de cooperação entre as empresas) e por uma orientação estratégica clara como postulado originalmente por Jordão (2011) e depois por Jordão et al. (2013). Esses aspectos foram centrais para o entendimento das práticas de GIC e também da criação de CI em todos seus elementos (capital humano, relacional e estrutural), sendo esse um dos achados surpreendentes da pesquisa, pois a análise de criação de CI não estava coberta no escopo inicial da pesquisa. De uma forma agregada e para aprofundar 0 entendimento das relações investigadas, a triangulação entre as informações obtidas nas entrevistas, documentos, observação direta e conversas informais permitiu constatar especificidades em algumas SMEs e nas relações entre elas. A 
triangulação indicou que: (i) a CAPA ficou parcialmente satisfeita e a GAMA não ficou satisfeita com os resultados alcançados depois da integração à rede; (ii) a insatisfação da GAMA advém mais de fatores externos, de anseios e particularidades do negócio, do que da rede propriamente dita; (iii) a rede promoveu melhorias nos indicadores de exportação de todas as empresas, inclusive da GAMA; e que (iv) esta empresa era uma das empresas mais avançadas sob o prisma dos negócios internacionais e já estava pronta para exportar desde o início do projeto, gerando uma ansiedade, um descontentamento e uma sensível perda de identidade com o grupo - que estava buscando se capacitar para atuar no comércio exterior carecendo de todo tipo de informações e incentivos. Esses resultados ampliam as observações de Andersen e Buvik (2002), de Freeman et al. (2006), permitindo inferir que o grau de maturidade da empresa interfere nas práticas de GIC em redes de SMEs. Além disso, a triangulação revelou que: (v) a percepção do CEO da GAMA contrasta a do CEO das demais, especialmente da ÔMEGA e SIGMA; (vi) os CEOs da ALFA, CAPA, DELTA, SIGMA e ZETA indicaram que a quantidade e a forma dos convênios foi inadequada (e o da ÔMEGA que é quase inexistente) para ajudar e fornecer apoio gerencial e suportar e fornecer alternativas para que as empresas possam fomentar e reter informações e conhecimentos necessários aos seus processos, especialmente o de internacionalização; e que (vii) todos os executivos tiveram o projeto como um meio de aprimorar e desenvolver certos conhecimentos que, em sua opinião, seriam muito mais difíceis de serem alcançados sem o suporte técnico e gerencial recebido do SEBRAE-ES.

Tomados em conjunto, os resultados mostram que no setor de indústrias em geral, a empresa ALFA mostrou-se a mais realizada com os resultados do projeto $\mathrm{e}$, no setor de agroindústrias, a empresa mais realizada foi a Ômega. A menos satisfeita deste setor foi a CAPA; e a empresa menos satisfeita daquele e de toda a rede foi a GAMA, como já discutido. De todas as SMEs, contudo, a empresa BETA foi a que mais cresceu (já sendo de médio porte) e a DELTA foi a que conseguiu alavancar mais e melhores oportunidade de internacionalização, inclusive com a criação de duas subsidiárias internacionais (com negócios correlatos) em dois diferentes continentes. A visão dos executivos sobre as práticas de GIC, em geral, e sobre a GC, em particular, revela que a integração à rede foi uma excelente oportunidade de crescimento e de negócios para as empresas e pessoas que nelas trabalham. Em geral, a visão do CEO da ÔMEGA foi a mais positiva de todos os entrevistados, em todos os aspectos analisados (salvo a questão das parcerias já discutida). Esse executivo, em particular, se mostrou bastante satisfeito com os resultados alcançados pelo projeto e por sua empresa ao longo dos últimos anos, ressaltando que não só compartilhou informações e conhecimentos, como também buscou colocar em prática os aprendizados recebidos através de ensinamentos teóricos e vivências práticas destacando que a disponibilidade em compartilhar informações, 
experiências e saberes mostra que os empresários do mesmo setor podem não ser somente concorrentes, mas também parceiros.

\section{Considerações finais}

A GIC tem sido tema de diversos estudos, sendo o conhecimento usualmente reconhecido pela literatura como um fator-chave à competitividade das empresas. O objetivo da pesquisa descrita neste artigo consistiu em analisar a influência da organização em rede sobre as práticas de GIC em SMEs industriais brasileiras.

Os resultados observados indicaram que todas as SMEs analisadas obtiveram melhorias (em processos, sistemas, produtos e/ou relacionamentos), em maior ou menor grau, decorrentes direta ou indiretamente de práticas de GIC depois da integração delas à rede. Foi possível perceber que os CEOs dessas empresas têm consciência dos pontos fortes e fracos, das habilidades e competências, bem como do papel da rede para o sucesso dos empreendimentos no contexto internacional. Constatou-se que muitos conhecimentos e vivências transmitidos não foram mapeados e internalizados, tornando-se explícitos - carecendo, portanto, de maior sistematização e formalização, mas houve certo consenso sobre os benefícios potenciais da partilha de informações e experiências entre as SMEs. Os entrevistados também acreditam que as empresas absorveram uma parte significativa do conhecimento construído na rede durante o projeto e que as interações e os relacionamentos interpessoais e interorganizacionais existentes dentro e fora das empresas e da rede foram centrais no processo de absorção de parte dos conhecimentos tácitos e de ampliação do saber explícito nas SMEs nos dois setores.

Os empresários acreditam que as empresas da rede até conhecem alguns processos de negócios das outras empresas, aproveitando do intercâmbio de informações sobre as especificidades dos processos industriais e comerciais umas das outras, mas, metade deles entendeu que esse aspecto não se deu em quantidade e de maneira suficiente, carecendo de ser aperfeiçoado. Os níveis de imersão na rede, de maturidade para exportar e capacidade de absorção das práticas compartilhadas variou entre as empresas analisadas, sendo fatores influenciadores das práticas de compartilhamento e sistematização de informações e saberes.

As visões gerais sobre o projeto foram variadas, mas, em geral, positivas. Apenas uma empresa revelou não estar satisfeita com os resultados do projeto, embora dele tenha se beneficiado. Entretanto, as metodologias de trabalho, as formas de condução dos grupos, a atividade de transmissão e compartilhamento de informações e conhecimentos, a maneira de divisão dos grupos e a organização das SMEs em rede foram questões apontadas como aquelas que apresentam maior necessidade de melhorias, especialmente no setor agroindustrial. As parcerias com instituições de ensino e pesquisa foram consideradas incipientes, 
carecendo de serem ampliadas e de que haja maior interação entre os atores envolvidos nos dois setores analisados. Em geral, sete dos oito executivos verificaram o fato de que a rede possibilitou diferenciais competitivos para as empresas participantes, gerando, desse modo, os conhecimentos necessários para o processo de exportação. De um modo especial, foi ressaltada a importância da criação de um 'Ba' que colabore para que as empresas e pessoas possam reter os conhecimentos adquiridos e/ou desenvolvidos no processo, sendo a cultura de cooperação considerada essencial nesse sentido. Os executivos acreditam que há bastante espaço para melhoria nesse quesito, ressaltando, todavia, que a própria organização em rede consiste em um espaço (contexto capacitante) para criação e compartilhamento de informações, experiências e saberes.

Os resultados, embora não indiscriminadamente generalizáveis, indicam que: (i) esse ' $B a^{\prime}$ ' já foi desenvolvido na maioria absoluta das empresas da rede (exceto em uma); (ii) existem ferramentas formais que ajudam a divulgar as práticas bem sucedidas de exportação dentro da rede, embora isso ainda precise ser melhorado; (iii) a rede possibilitou diferenciais competitivos para as SMEs dos dois setores; (iv) a rede foi fundamental no processo de desenvolvimento dos conhecimentos e das competências necessárias para a exportação (em menor grau em duas empresas); (v) as informações são relativamente partilhadas; (vi) culturas similares, linguagem e experiência compartilhadas podem ajudar a criar e difundir o saber no interior da rede. Adicionalmente, constatou-se que ainda há muito a se fazer para que as SMEs possam, de fato, criar, armazenar, adquirir, desenvolver, transmitir e compartilhar as informações e os conhecimentos produzidos em suas empresas e, ainda mais, para que isto se dê de forma efetiva no interior da rede. Não obstante, a própria articulação em rede permitiu que todas as empresas, sem exceção, pudessem usufruir e desenvolver práticas de GIC - de um modo que não teriam condições de fazer sem estarem associadas em rede. Isso porque essas práticas ajudaram tanto no processo de desenvolvimento das competências, quanto na partilha de experiências e na criação de uma cultura própria no interior do grupo. De fato, o ambiente em rede formou um ' $B a^{\prime}$ ' intraorganizacional, que foi percebido como fundamental para estimular a GIC nas SMEs. De modo mais claro e enfático constatou-se que a absorção de conhecimentos teóricos e a partilha de experiências ocorrem de forma mais vinculada às empresas do que à rede e que o envolvimento das pessoas (a começar pelo CEO) é fundamental para o sucesso das práticas de GIC no âmbito das redes. Isso porque $o$ processo de partilha de informações e de fomento à aprendizagem organizacional pode ser percebido, realizado e trabalhado pelas empresas pertencente à rede de maneiras diferentes, indo além do que foi observado na literatura anterior. Ao realizar uma análise comparativa entre o setor agroindustrial e o de indústrias em geral, observou-se que não foram constatadas diferenças significativas entre os mesmos em relação à GIC. Adicionalmente, foi possível perceber que 
tanto a introdução de novas práticas de criação, sistematização e partilha de informações e conhecimentos, quanto à melhoria das práticas já existentes não foram afetadas pelo setor em que as SMEs atuam, mas, sim, pelo grau de imersão à rede. Além disso, constatou-se, em ambos os setores, que a cultura organizacional (de cooperação entre as empresas) e uma orientação estratégica clara e definida foram aspectos centrais para o entendimento das práticas de GC e, surpreendentemente, ajudaram a entender a criação de CI observada por incrementos no capital humano, estrutural e relacional.

Finalmente, vale dizer que, embora se tenha percebido que a rede consistiu em um meio de criar, aprimorar, estimular e desenvolver informações e conhecimentos que seriam muito mais difíceis de serem desenvolvidos, ampliados ou sistematizados sem a mesma, há ainda a necessidade de mais estudos sobre o tema, seja na forma de casos comparados, setoriais, ou através de novas metodologias/outros contextos para colaborar para um maior entendimento tanto da GIC quanto do $\mathrm{CI}$ nesse contexto, principalmente se levarmos em conta a relevância estratégica das SMEs para a economia e a sociedade; e o papel da GIC para essas empresas.

\section{Referência}

AMAL, M.; FREITAG FILHO, A. R.; MIRANDA, C. M. S. Algumas evidências sobre o papel das redes de relacionamento e empreendedorismo na internacionalização das pequenas e médias empresas. Revista Faces de Administração Journal, Belo Horizonte, v. 7, n. 1, p. 63-80, 2008.

ANDERSEN, O.; BUVIK, A. Firm's internationalization and alternative approaches to the international customer/market selection. International Business Review, v. 11, n. 3, p. 347-363, 2002.

BARDIN, L. Análise de Conteúdo. 3. ed. Lisboa: Edições 70, 221p., 2004.

BARROS, F. S. O.; PONTE, V. M. R.; FARIAS, I. Q. Gerenciamento do capital intelectual: um estudo em empresas do setor têxtil cearense. BASE - Revista de Administração e Contabilidade da UNISINOS, v. 4, n. 2, p. 137-148, 2007.

BEHR, R. R.; NASCIMENTO, S. P. A. Gestão do conhecimento como técnica de controle: uma abordagem crítica da conversão do conhecimento tácito em explícito. Cadernos EBAPE - FGV, v. 1, n. 1, p. 1$11,2008$.

CALDAS, P. T.; CANDIDO, G. A. Inter-Organizational Knowledge Conversion and Innovative Capacity in Cooperative Networks. Journal of Technology Management \& Innovation, v 8. S.I., p. 104-114. 2013.

CASTELLS, M. A sociedade em rede. A era da informação: economia, sociedade e cultura. São Paulo: Paz e Terra. 1999. 
CHOO, C. W.; ALVARENGA NETO R. C. D. Beyond the Ba: managing enabling contexts in knowledge organizations. Journal of Knowledge Management, v. 14, n. 4, p. 592-610, 2010.

COOPER, D. R.; SCHINDLER, P. S. Business research methods. 9th ed. New York: McGraw Hill-Irwin, 2006.

DAVENPORT, T. H.; PRUSAK, L. Conhecimento empresarial: como as organizações gerenciam o seu capital intelectual. Rio de Janeiro: Campus. 1998.

DURST, S.; EDVARDSSON, I. R. Knowledge management in SMEs: a literature review. Journal of Knowledge Management, v. 16, n. 6, p. 879903. 2012.

EISENHARDT, K. Building theories from case study research. Academy of Management Review, v. 14, n. 14, p. 532-500, 1989.

FREEMAN, S.; EDWARDS, R.; SCHRODER, B. How smaller born global firms use networks and alliances to overcome constraints to rapid internationalization. Journal of International Marketing, Chicago, v. 14, n. 3, p. 33-63, 2006.

GEORGE, A.; BENNETT, G. Case studies and theory development in the social sciences. Cambridge, Massachusetts: MIT Press, 2005.

GRANOVETER, M. Economic action and social structure: the problem of embeddedness. American Journal of Sociology, Chicago, v. 91, n. 3, p 481-510, 1985.

GRANT, R. Toward a knowledge based theory of the firm. Strategic Management Journal, v. 50, n. 17, p. 109-122, Winter 1996.

HOSS, O. Modelo de avaliação de ativos intangíveis para instituições de ensino superior privado. 170f. Tese (Doutorado em Engenharia de Produção) - UFSC, Florianópolis, 2003.

JARDON, C. M. F., MARTOS M. S. Capital intelectual y competencias distintivas en PYMEs madereras de argentina. Revista de Administração de Empresas (RAE), v. 54, n. 6, p. 634-646. 2014.

JARDON, C. M; MARTOS, M. S. Intellectual capital as competitive advantage in emerging clusters in Latin America. Journal of Intellectual Capital, v. 13, n. 4, p. 462-481. 2012.

JICK, T. Mixing qualitative and quantitative methods: triangulation in action. Administrative Science Quarterly, v. 24, p. 602- 610, 1979.

JORDÃO, R. V. D. . Controle do capital intelectual: um modelo aplicado à gestão dos ativos do conhecimento. In: Anais do II Congresso Nacional de Administração e Ciências Contábeis - AdCont 2011, Rio de Janeiro. 1 CD Rom.

JORDÃO, R. V. D.. Strategic relationships between theory of the firm, mergers \& acquisitions and management control systems. In: Anais do 
8th Iberoamerican Academy Conference - IAM 2013, São Paulo. 1 CD Rom.

JORDÃO, R. V. D.; NOVAS, J. C.. A Study on the use of the balanced scorecard for strategy implementation in a large Brazilian mixed economy company. Journal of Technology Management \& Innovation, v. 8, p. 98107, 2013.

JORDÃO, R. V. D.; NOVAS, J. C.; Souza, A. A.; NEVES, J. T. R.. Controle do capital intelectual: um modelo aplicado à gestão dos ativos do conhecimento. Revista Iberoamericana de Estratégia, v. 12, p. 195-227, 2013.

JORDÃO, R. V. D.; PELEGRINI, F. G.; JORDÃO, A. C. T. Fatores críticos na gestão de projetos: um estudo de caso numa grande empresa latinoamericana de classe mundial. Gestão \& Produção (UFSCAR. Impresso), v. 22, p. 280-294, 2015.

JORDÃO, R. V. D.; SOUZA, A. A.. Company's acquisition as a factor of change on the management control system: a strategic analysis from the perspective of the contingency theory. Revista Universo Contábil, v. 9, p. 75-103, 2013.

JORDÃO, R. V. D.; COLAUTO, R. D.. Evidenciação voluntária de informações contábeis: um estudo empírico sobre a divulgação do capital intelectual em instituições financeiras. Revista Portuguesa e Brasileira de Gestão (Lisboa), v. 12, p. 30-43, 2013.

JORDÃO, R. V. D.; SOUZA, A. A.; AVELAR, E. A. Organizational culture and post-acquisition changes in management control systems: An analysis of a successful Brazilian case. Journal of Business Research, v. 67, p. 542549, 2014.

KHALIQUE, M.; BONTIS, N. SHAARI, J. A. N. B.; ISA, A. H. M. Intellectual capital in small and medium enterprises in Pakistan. Journal of Intellectual Capital, v. 16, n. 1, p. 224-238. 2015.

KHALIQUE, M.; ISA, A. H. B. Md., Intellectual Capital in SMEs Operating in Boutique Sector in Kuching, Malaysia. The IUP Journal of Management Research, v. 13, n. 2, p. 17-28. 2014.

LEI, D.; HITT, M.A.; BETTIS, R. Dynamic core competences through metalearning and strategic context. Journal of Management, v. 22, n. 4, p. 549-569, 1996.

MOURITSEN, J. et al. Developing and managing knowledge through intellectual capital statements. Journal of Intellectual Capital, v. 3, n. 1, p.10-29, 2002.

NGAH, R.; IBRAHIM, A. R.. The Relationship of Intellectual Capital, Innova tion and OrganizationalPerformance: A Preliminary Study in Malaysian SMEs. International Journal of Management Innovation Systems, v. 1, n. 1, p. 1-13. 2009. 
NONAKA, I.; NISHIGUCHI, T. Knowledge emergence. New York: Oxford, 2001.

NONAKA, I.; TOYAMA, R.; KONNO, N. SECI, Ba and leadership: a unified model of dynamic knowledge creation. Long Range Planning, v. 33, n. 1, p. 5-34. 2000.

PELUFÊ, M. S. O uso de informação do ambiente organizacional por indústrias de setor metal-mecânico de micro e pequeno portes da região de Passo Fundo, RS. 71f. Dissertação (Mestrado em Ciência da Informação) - UFMG, Belo Horizonte, 2005.

PRAHALAD, C.K.; HAMEL, G. The core competence of the corporation. Harvard Business Review, Boston, v. 68, n. 3, p. 79-91, maio/jun. 1990.

RICHARDSON, R. J. Pesquisa social: métodos e técnicas. 3. ed. São Paulo: Atlas. 1999.

ROMITI, A.; SARTI, D.. Governance of Networks of Small Enterprises: A Knowledge Perspective - Some Case Studies in the Mechanical Industry in Italy. Anais do 30 European Conference on Intellectual Capital. University of Nicosia, Cyprus. 2011.

SABEL, C. Moebius-strip organizations and open labor markets: some consequences of the reintegration of conception and execution in a volatile economy. In: COLEMAN, J.; BOURDIEU, P. Social theory for a changing society. Boulder: Westview Press, p. 23-54, 1991.

SERVIÇO BRASILEIRO DE APOIO ÀS MICRO E PEQUENAS NO ESPÍRITO SANTO (SEBRAE-ES). Série Cooperação Internacional. Ed. SEBRAE. 2011. 1 CD-ROM.

SILVEIRA, P. A. ; ALPERSTEDT, G. D. O processo de internacionalização de uma empresa de pequeno porte do setor moveleiro do oeste de Santa Catarina sob a ótica do empreendedor. In: Anais do III Encontro de Estudos em Estratégia - 3ES, São Paulo, 2007. 1 CD-ROM.

SPENCER, J. W. Firms' knowledge-sharing strategies in the global innovation system: empirical evidence from the flat panel display industry. Strategic Management Journal, v. 24, n. 3, p. 217-33, 2003.

SUCHMAN, L. Plans and situated actions: the problem of human-machine communication. New York: Cambridge University Press, 1987.

TOMAÉL, M. I. Redes de conhecimento: o compartilhamento da informação e do conhecimento em consórcio de exportação do setor moveleiro. 2005. 289f. Tese (Doutorado em Ciência da Informação) UFMG, Belo Horizonte, 2005.

VALKOKARI, K; HELANDER, N. Knowledge management in different types of strategic SME networks. Management Research News, v. 30, n.8, p. 597-608. 2007.

YIN, R. Case study research: design and methods. Beverly Hills, CA: Sage Publishing, 1984. 